\title{
GRANDMASTERS VS. COMPUTERS: LATEST
}

\author{
Jan Louwman
}

\section{Rotterdam}

The last few months witnessed two Grandmasters in a simultaneous exhibition against chess computers. The Rotterdam protagonist was the Russian David Bronstein, while in the village of Dieren, the well-known Dutch Grandmaster Paul van der Sterren played the leading role. Allowing for Bronstein's advanced age of 66 years, it was decided to have him oppose 16 computers, rather less than is usual nowadays. The reasoning behind this is that even micros nowadays are quite capable and it may be held that a Grandmaster may be harder put to it to oppose simultaneous computers than to compete with the better brand of club players. The background to this is that computers are not imposed upon by their opponent's being Grandmaster, since they are not aware of the identity of their adversary. To exacerbate matters, computers commit few errors, so many hours may elapse before a game is decided.

In spite of all this, Bronstein achieved a signal victory against his chip-based opponents. He vanguished eleven games, drew three times and lost only twice. Even by present-day standards, 78-percentage score in computer simultaneous play is a respectable achievement and many a game showed Bronstein to be equal to his achievements in his best years ever. The programs he lost to were Mephisto Portorose 68030 and the CXG Dominator, whereas his draws were against the Mephisto Portorose 68020, Mephisto Polgar $10 \mathrm{MHz}$ and the American Program M Chess, formerly known as AI Chess, which latter exploited a PC with 386xx hardware. The last-mentioned program, due to US programmer Martin Hirsch, is a regular competitor in tournaments in the Netherlands, i.e., a member of computer teams or an opponent to strongly-playing human adversaries. It is an excellent chess program, the more so if it enjoys the advantage of fast hardware.

It was 4.5. hours only before all games were finished and the good score achieved by Bronstein can only be interpreted as a major achievement by this Soviet Grandmaster.

A few weeks later, one of the better Dutch Grandmasters, Paul van der Sterren, was due to test his strength against 24 chess computers. Readers please note that van der Sterren had meanwhile achieved a rating far exceeding Bronstein's. The larger number of opponents and the sweltering heat prevailing did not exactly facilitate van der Sterren's task. It took him all of 8.5 hours of playing to finish his simultaneous performance and even then he only achieved a 56 percentage. Paul won eight, drew eleven and lost five. Signally predominant among his opponents were ten entries by programmer Ed Schröder, participating in the tourney for the respectable total of 6.5 . We are happy to publish, selected and annotated by Jeroen Noomen, a few games appropriately culled.

Switching to a subject beyond the tournaments, we have the "quicky" record of Mephisto Portorose 68030 against (very) strong chess-players, who averaged 2090 on the FIDE rating scale. In all, 118 five-minute speedchess games were played against these humans. The computer won 103, drew 11, and lost only 4 . This makes for a percentage of 92 , imposing and frightening both. One should, however, allow for the known circumstance that this computer system is known to be almost indecently good at speed chess. If this needs substantiation, let us mention that this computer recently achieved first place in the FRG's official speed-chess championship with a large lead to its strong flesh-and-blood competitors. The moral is that micros, too, nowadays should be taken seriously.

\section{White: Bronstein \\ Black: Portorose 68030}

1. d4 d5 2. c4 c6 3. Nf3 Nf6 4. Nc3 dxc4 5. a4 Na6 6. e3 Bg4 7. Bxc4 e6 8. 0-0 Nb4 9. a5!? So far Portorose's book. White's last move prevents Black moving a5, which would result in the b4-square falling into Black's hands. 9. ... a6 10. Be2 Bf5 11. Bc4 Be7 12. Ne5 0-0 13. Qf3 Nd7 14. Nxd7 Qxd7 15. Rdl Bc2 16. Rd2 Qc7 17. e4 Bg5 18. Re2 Bf6 19. e5 Be7 Black has lost quite some tempi by his bishop moves. As against this, d4 has lost some of its strength. 20. Be3 Rad8 21. Ne4 c5! A standard response for the position given. 22. dxc5 Rd1+ 23. Re1 Rxa1 24. Rxa1 Qxe5 25. Nd6 Bxd6 26. cxd6 Qxb2 27. Rf1 Black is a Pawn up, though the pair of Bishops and the passed Pawn on d6 tend to compensate this. 27. ... Qc3 28. Qg4 Nc6 29. Be2 Rd8 30. h4 Since 
the Pawn on d6 is doomed, Bronstein in despair attempts an attack: 30.... Rxd6 31. h5 Nxa5? Much too greedy. The a-Pawn will keep. All of a sudden, White is granted strong attacking chances. 32. h6 g6? Better was f5. 33 . Rc1 Nb3 34. Qf4? The decisive error. Günther Löwenthal had indicated 34. Rxc2! Qxc2 and only then 35. Qf4; doing this White might have won. 34. ... Nxc1 35. Qxd6 Nxe2+ 36. Kh2 Qc8 37. Bg5 Bb3 38. Qe5 f6 39. Qxf6 Qc7 40. f4 Bd5 41. g3 Nd4! 42. Kh3 Nf3 43. f5 Nxg5+ 44. Qxg5 exf5 45. Qf6 a5 White resigns.

White: Bronstein

Black: The Final Chesscard

1. d4 e6 2. Nf3 d5 3. c4 Nf6 4. Nc3 Be7 5. Qc2!? An interesting variation, invented by Tony Miles. 5. ... 0-0 6. e4 dxe4 7. Nxe4 Nxe4 8. Qxe4 Nd7 9. Bd3 Nf6 10. Qe2 c5 The Final Chesscard was not led astray by White's unusual fifth move. The program will continue to move logically, here as in the sequel. 11. dxc5 Qa5+12. Bd2 Qxc5 13. Bc3 Rd8 14. 0-0 Bd7 15. Ne5 Rac8 16. Rad1 Ba4 17. b3 Bc6 Also possible was Be8. 18. b4 Qb6 19. a3 Ba4 20. Rc1 Qc7 21. Rfe1 Bf8 In order to prevent potential sacrifices on f7. 22. Bb1 b6 23. Qf3 a6 24. Qh3 Bronstein assidiously constructs an attack. 24. ... h6 25. Qe3 Qb7 26. h4! Since the centre is stabile, White is free to start an flanking attack. 26. ... Be7?! It seems correct to play Be8 to protect f7. Even so, the white attack is a threat. 27. g4! Ne8 allows the penetration as below, though nothing better springs to mind. 28. Nxf7! Kxf7 29. Qxe6+ Kf8 30. Bh7 and after Nf6 follows Bxf6 and mate. Black resigns.

White: Paul van der Sterren

Black: M Chess

1. e4 c5 2. Nf3 d6 3. d4 cxd4 4. Nxd4 Nf6 5. Nc3 Nc6 6. Bg5 e6 7. Qd2 Be7 8. 0-0-0 0-0 9. f3 Usual is 9. f4. 9. ... d5 10. exd5 Nxd5 11. Bxe7 Qxe7 Ncxe7 would have been more consequent. 12. Nxd5 exd5 13. Kb1 Bd7 14. Nb3 Be6 15. Bb5 a6 16. Bxc6 bxc6 17. Qd4 White goes for controlling the black squares. 17. ... Rab8 18. Rhe1 Rfe8 19. Re5 Rb4 20. Qc3 Rc4 21. Qe1 Rb8 22. Nd4 Qa3 23. Nb3 Qd6 24. Qg3 Qb4 25. c3 Qd6 26. Rd2 a5 27. Nd4 Rxc3 28. Nxe6 fxe6 29. Rxe6 Rc4 30. Re7 Qc5 Threatening Qg1+. 31. Re1 Qb6 M Chess is prepared for a draw by repetition of moves, but Van der Sterren still has something at his sleaves. 32. a3 Qd8 33. Rde2 a4 34. Re7 Apparently, Black is in a bad position, but the American program succeeds in finding an enchanting combination leading to a draw. 34. ... Rxb2+! Because 35. Kxb2 Qb6 is a dead loss for White. 35 . Ka1! Rb1!! After 36. Rxb1 follows Qxe7 and after 36. Kxb1 Qb6 looses. So White must repeat moves, eventually leading to a draw. 36. Ka2! Rb2! Draw.

White: Paul van der Sterren

Black: Mephisto College Turbo

1. d4 d5 2. c4 dxc4 3. Nf3 Nf6 4. Nc3 a6 5. e4 b5 6. e5 Nd5 7. a4 Nxc3 8. bxc3 c6 9. Ng5 h6 10. Ne4 Qd5 11. Qf3 e6 12. Be2 Nd7 13. 0-0 Bb7 14. Qg4! prevents ... Be7 and threatens 15 . Bf3 followed by Nf6. 14. ... bxa4?! Not an elegant move. Van der Sterren refutes the computer's materialism in this game. 15. Bf3 Qa5 16. Ba3 Bxa3 17. Qxg7! Ke7 Threatens Rag8. 18. Bh5! Bb2? A quick loss. Better were 18. ... Raf8. 19. Bxf7 Bxa1 20. Nd6! With all its terrible threats. A despairing move is yet to follow. 20.... Rh7 21. Qxh7 Black resigns.

White: Paul van der Sterren

Black: Mephisto Polgar $10 \mathrm{MHz}$

1. d4 Nf6 2. c4 e6 3. Nf3 b6 4. g3 Ba6 5. b3 Bb4+ 6. Bd2 Be7 7. Bg2 d5 8. cxd5 exd5 9. 0-0 c5 10. Nc3 Nc6 11. Bf4 Ne4 12. Re1 0-0 A typical Queens Indian position has arisen. Should Black avail himself of the $\mathrm{d} 4 \mathrm{ex}-$ change, he will always gain an isolated d-Pawn, whereas White by playing $\mathrm{dxc} 5$ concedes to it that Black is saddled with hung Pawns on $\mathrm{d} 5$ or c5. Rather than this, Black prefers an active line of play. 13. dxc5 Nxc3 14. Rxc3 bxc5 15. Ne1 Nd4 16. Nd3 Qa5 17. Bd2 Qxa2 18. Bxd5 Rad8 Clearly, White has not done too well, all the initiative is with Black and the white pieces are standing around somewhat awkwardly. 19. Bc4 Bxc4 20. bxc4 Bf6! 21. Re1 The decisive error, due to lose the exchange. 21. ... Nxe2+! 22. Rxe2 Bxc3 23. Bxc3 Qxc4 24. Qa1 Qxd3 25. Rd2 Qf3 White resigns. 